\title{
Analogies between elastic and capillary interfaces*
}

\author{
Jacco H. Snoeijer \\ Physics of Fluids Group, Faculty of Science and Technology, MESA + Institute, University of Twente, \\ 7500 AE Enschede, The Netherlands \\ and Mesoscopic Transport Phenomena, Eindhoven University of Technology, \\ Den Dolech 2, 5612 AZ Eindhoven, The Netherlands
}

(Received 16 July 2016; published 18 October 2016)

\begin{abstract}
In this paper we exploit some analogies between flows near capillary interfaces and near elastic interfaces. We first consider the elastohydrodynamics of a ball bearing and the motion of a gas bubble inside a thin channel. It is shown that there is a strong analogy between these two lubrication problems, and the respective scaling laws are derived side by side. Subsequently, the paper focuses on the limit where the involved elastic interfaces become extremely soft. It is shown that soft gels and elastomers, like liquids, can be shaped by their surface tension. We highlight some recent advances on this class of elastocapillary phenomena.
\end{abstract}

DOI: 10.1103/PhysRevFluids.1.060506

\section{INTRODUCTION}

Capillary flows and wetting phenomena appear naturally in a broad variety of contexts that involve droplets, thin films, and liquid jets. A challenging aspect in resolving such flows is that the shape of the free surface is not known a priori, but has to be found self-consistently with the flow. Owing to surface tension, the normal stress is determined by the curvature of the interface, which together with the kinematic boundary condition gives a nonlinear coupling to the flow. As a consequence, even viscosity-dominated flows becomes highly nonlinear; this is exemplified explicitly in the nonlinear lubrication (thin film) equation. Similar free boundary problems arise in the context of fluid-structure interaction or elastohydrodynamics, where flow induces elastic deformation of the confining boundaries. Examples can be found on all length scales, ranging from airfoil flutter, wind-deformed trees, and flow in collapsible tubes down to cellular motion through the beating of flexible flagella. In all cases, the shapes of the elastic bodies have to be found self-consistently with the flow, coupled kinematically and by elastic stresses.

Though liquid and elastic responses to shape deformation are very different, the structure of the resulting flows can be very similar. This is illustrated in Fig. 1, comparing an image of liquid drop impact (left panel [1]) and the approach of a rigid sphere above a soft elastic layer (right panel [2]). In both cases, a squeeze flow appears in the thin gap between the impactor and the substrate; this leads to a buildup of a lubrication pressure that is able to deform the droplet and the elastic layer [1-7].

In the first part of this paper we will fully work out the analogy between capillary and elastic flows for two closely related problems: the motion of a bubble in a narrow channel [Bretherton's problem, Fig. 2(a)] and the elastohydrodynamic lubrication appearing in deformable ball bearings [Fig. 2(c)]. The former is a paradigmatic example for capillary flows [8-11], while the latter is classical in the context of tribology [12-16]. Here we show that both flows exhibit the same mathematical structure and we will derive the resulting scaling laws using the very same method in both cases.

Elastic lubrication phenomena are not restricted to the context of very stiff bearings, but are also encountered for rubber seals or for very soft (bio)materials such as articular cartilage. Interestingly, when considering exceedingly soft matter, the wetting and lubrication becomes even more intricate

*This paper is based on an invited lecture given by Jacco H. Snoeijer at the 68th Annual Meeting of the APS Division of Fluid Dynamics, which was held 22-24 November 2015 in Boston (MA), USA. 


\section{JACCO H. SNOEIJER}
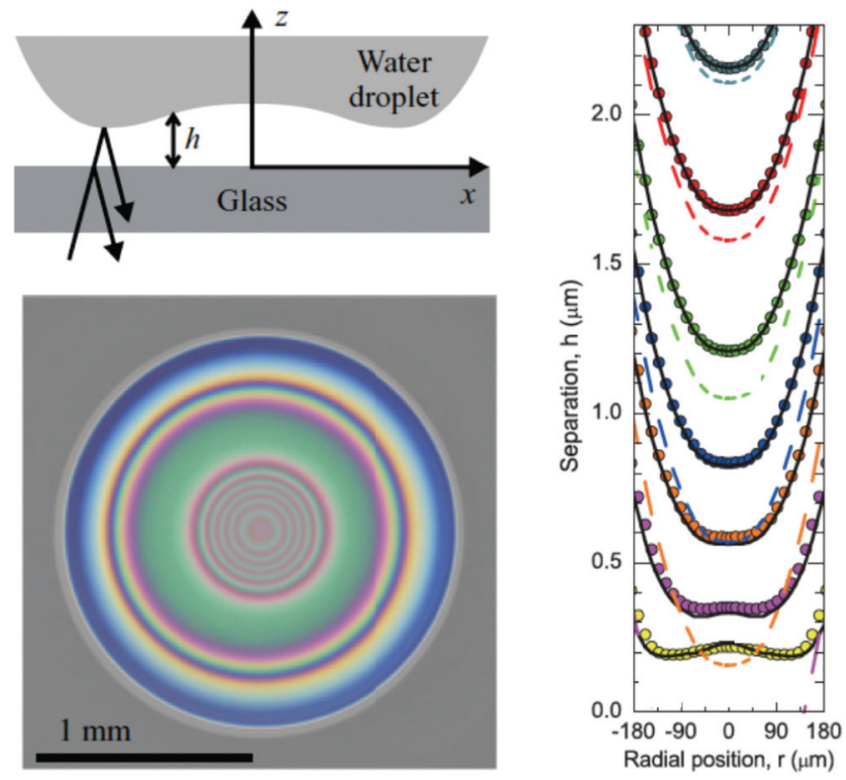

FIG. 1. Dimple formation in fluid and elastic interfaces. Shown on the left is a dimple below an impacting liquid drop. The air between the drop and the rigid wall is squeezed out of the thin gap and the resulting lubrication pressure induces a dimple measured by interferometry. (Reprinted with permission from [1]. Copyright 2012 by the American Physical Society.) On the right are very similar dimple profiles observed when a rigid sphere approaches an elastically compliant substrate. (Reprinted with permission from [2]. Copyright 2015 by the American Physical Society.)

and the analogy with liquid interfaces takes on a whole new meaning. Namely, gels and elastomers themselves possess a solid surface tension $\gamma_{s}$ and as a consequence share common mechanical features with liquid interfaces [17-22]. Indeed, surface effects can compete with, and even dominate over, bulk elastic effects when Young's modulus $E$ of the solid is sufficiently small. More precisely, one can define the ratio of solid surface tension over Young's modulus $\gamma_{s} / E$ as the elastocapillary length. This length sets the scale below which capillary effects of the solid dominate over its bulk elasticity. For common soft gels with moduli in the range of $1-100 \mathrm{kPa}$, this length $\gamma_{s} / E$ can become as large as $1-100 \mu \mathrm{m}$ and leads to a new class of elastocapillary phenomena that are

(a)

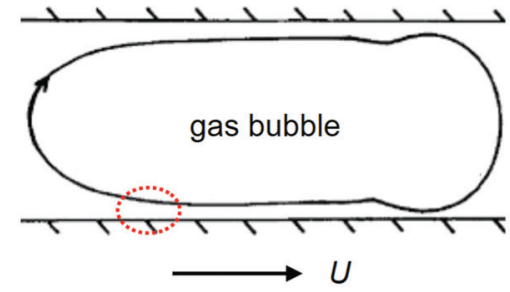

(b)

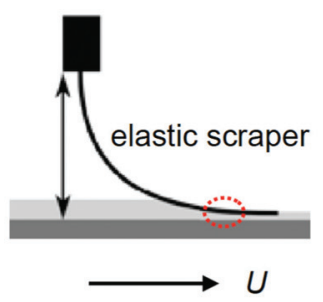

(c)

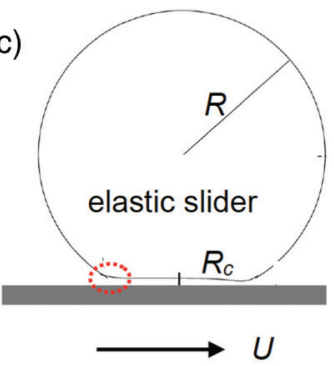

FIG. 2. Three similar lubrication problems. (a) Long bubble moving inside a channel [8]. (Adapted from [8], reproduced with permission from Cambridge University Press.) (b) Flexible plate that leaves a thin liquid film [23]. (Adapted from [23], reproduced with permission from Cambridge University Press.) (c) Sliding Hertzian contact $[12,16]$. (Reproduced from [16] with the permission of AIP Publishing.) The red dashed zone in all three panels indicates the inner region of height $H$ and length $L$, which is described by a lubrication flow. 


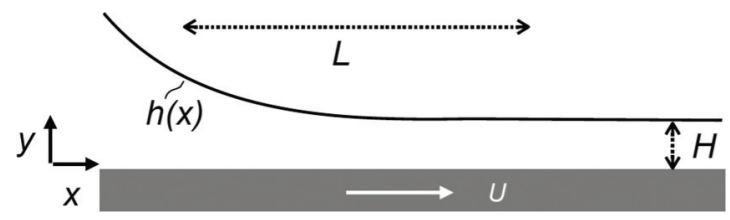

FIG. 3. Zoom of the lubrication zone, shown as the dashed regions in Fig. 2. This inner region has a horizontal scale $L$ and a vertical scale $H$. In the frame comoving with the deformable interface, denoted by $h(x)$, the wall is moving with velocity $U$.

currently being actively explored [20-22]. In the second part of the paper we therefore highlight some striking features of elastocapillary interfaces and discuss some deep connections between between the adhesion of solids and the wetting of liquids.

\section{LUBRICATION OF BUBBLES AND SOLIDS}

To illustrate the analogy between capillary lubrication and elastic lubrication, we will focus on two prototypical problems: Bretherton's analysis of long bubbles in a narrow tube [Fig. 2(a)] and the elastohydrodynamic lubrication in tribology [represented here by a soft slider in Fig. 2(c)]. Both cases involve the steady motion of a deformable object parallel to a rigid wall, with a prescribed velocity $U$, lubricated by a thin liquid film of thickness $H$. A third example that exhibits a similar structure is that of the flexible scraper that leaves behind a thin liquid film [Fig. 2(b)].

The question we address for all cases in Fig. 2 is how the thickness of the lubrication film scales with the sliding velocity, i.e., $H \sim U^{\alpha}$. Though the three lubrication flows exhibit different scaling exponents $\alpha$, the structure of the problems is strictly identical. A key ingredient is that the lubrication film becomes asymptotically thin at low velocities, so the hydrodynamics affects only a small region; this lubrication zone is indicated by the red dashed circles in Fig. 2. The height $H$ and width $L$ of this hydrodynamic zone are unknown a priori and have to be determined self-consistently. At larger scales, the elastic or capillary interfaces are essentially at equilibrium. The purpose of this section is to highlight the equivalent structure of these elastic and capillary lubrication flows. We work out the three cases in the form of scaling analysis; the full solution involves a more detailed matched asymptotic expansion based on similarity solutions, for which we refer to [8,16,23].

\section{A. Bretherton bubbles: $\alpha=2 / 3$}

We first focus on the flow in the thin film below the bubble, for which a more detailed zoom is given in Fig. 3. The flow can be solved by the Stokes equation, which in the lubrication approximation reduces to

$$
\frac{d p}{d x}=\eta \frac{d^{2} v_{x}}{d y^{2}}
$$

The horizontal fluid velocity naturally scales as $v_{x} \sim U$, while the vertical coordinate is set by the film thickness $y \sim H$. However, the horizontal length scale $x \sim L$ and the pressure scale $p \sim P$ are still unknown and we therefore write (1) as

$$
\frac{P}{L} \sim \frac{\eta U}{H^{2}}
$$

(lubrication). The horizontal scale is sometimes referred to as the length of the dynamical meniscus [11].

A second equation results from the relation between pressure $p(x)$ inside the lubrication film and the shape of the interface $h(x)$. For capillary interfaces this is given by the Laplace pressure 


\section{JACCO H. SNOEIJER}

$p=-\gamma d^{2} h / d x^{2}$, which in terms of the scaling law gives

$$
P \sim \frac{\gamma H}{L^{2}}
$$

(deformation).

The two equations (2) and (3) do not yet fully specify the lubrication layer, since there are three unknown scales: the horizontal and vertical lengths $H$ and $L$, respectively, and the pressure scale $P$. The problem is closed by a matching condition that connects the small-scale lubrication region to the large-scale interface profile. The latter is essentially unaffected by the thin flowing layer, so on large scales the interface is at equilibrium. For the Bretherton problem, the meniscus has a curvature that is fixed by the channel size $R$. In order for the thin film region to match with this outer shape $h \sim x^{2} / R$, the lubrication film should satisfy a geometric matching condition

$$
H \sim \frac{L^{2}}{R},
$$

which provides the desired meniscus curvature $R^{-1}$.

The various scales can now be determined from Eqs. (2)-(4), e.g., by eliminating the pressure $P$ and the horizontal length $L$ and subsequently solving for $H$. This gives the classical scaling law

$$
H \sim R\left(\frac{\eta U}{\gamma}\right)^{2 / 3},
$$

hence $H \sim U^{2 / 3}$. The same exponent is also observed for the Landau-Levich-Derjaguin problem in dip coating [9] or for pulled soap films [10]. On the right-hand side one can recognize the capillary number $\mathrm{Ca} \equiv \eta U / \gamma$, so we introduce the more familiar form

$$
H=1.34 R \mathrm{Ca}^{2 / 3},
$$

where we now also provide the prefactor as obtained from the full matched asymptotics solution in [8].

It is important to note that the matching procedure relies on the assumption that the film thickness $H$ is small with respect to the macroscopic size $R$, so (6) implies that the analysis is valid for $\mathrm{Ca} \ll 1$. One further verifies that $L \sim R \mathrm{Ca}^{1 / 3}$, so for small capillary numbers one finds $H / L \ll 1$, justifying the lubrication assumption.

\section{B. Elasticity}

The exact same strategy can now be followed for the elastic problems of lubricated Hertz contacts and the flexible scraper. The lubrication equation (2) is the same, but differences appear in (3) and (4), respectively, describing the relation between pressure and deformation and the static interface shapes. Below we adapt these relations and derive the different scaling laws.

\section{A flexible scraper: $\alpha=3 / 4$}

For the scraper problem of Fig. 2(b) we closely follow Seiwert et al. [23]. They treat the scraper as a thin elastic plate of length $R$ and bending modulus $B=E t^{3} / 12\left(1-v^{2}\right)$, where $t$ is the plate thickness. The relation between pressure and the shape of the plate is $p=B d^{4} h / d x^{4}$, which leads to the scaling law

$$
P \sim \frac{B H}{L^{4}}
$$

(deformation). This replaces (3) in the bubble analysis.

The static outer shape in the experiment in [23] is imposed by clamping the scraper at one end. The other end of the scraper is assumed to approach the liquid film with free end conditions. This gives as a boundary condition that the internal torque of the beam vanishes and hence the scraper's 
curvature $d^{2} h / d x^{2} \rightarrow 0$ as it approaches the film. In addition, we will consider that the tip of the beam is tangent to the coated surface, i.e., $d h / d x \rightarrow 0$. Hence the local shape is of the form $h \sim x^{3} / R^{2}$, where the length scale of the outer solution is set by the plate length $R$. Once more, expressing the outer shape in terms of the horizontal scale of the inner solution $x \sim L$, we obtain a matching condition

$$
H \sim \frac{L^{3}}{R^{2}}
$$

replacing (4) in the bubble analysis.

The final step is to eliminate $L$ and $P$ between (2), (7), and (8), which yields the flowing thickness below the scraper

$$
H=0.66 R\left(\frac{\eta U R^{2}}{B}\right)^{3 / 4} .
$$

Here we again we used the prefactor from the matched asymptotic expansion, ${ }^{1}$ as derived by Seiwert et al. [23]. We thus find a different scaling exponent as compared with the Bretherton problem, namely, $H \sim U^{3 / 4}$. However, it is clear that the solution structure is strictly identical.

\section{Hertz contacts: $\alpha=3 / 5$}

We now consider the elastic slider problem in Fig. 2(c), which is equivalent to the elastohydrodynamics of rotating ball bearings. First, the elastic cylinder of radius $R$ is pressed against a solid wall with a load $F$ (force per unit length). This is a two-dimensional version of a Hertz contact for which the width of the contact zone $R_{c}$, indicated in Fig. 2(c), can be computed as [13]

$$
R_{c}=R\left(\frac{4 F\left(1-v^{2}\right)}{\pi E R}\right)^{1 / 2} .
$$

Subsequently, the cylinder is set into motion with respect to the wall while lubricated by a thin film.

In this case of a bulk elastic material, the relation between pressure $p(x)$ and the gap thickness $h(x)$ is a bit more involved, namely,

$$
h(x)=c+\frac{x^{2}}{2 R}-\frac{2\left(1-v^{2}\right)}{\pi E} \int_{-\infty}^{\infty} d x^{\prime} p\left(x^{\prime}\right) \ln \left|x-x^{\prime}\right| .
$$

The first term on the right-hand side represents the shape of the cylinder in its reference state, i.e., without any elastic deformation. The integral represents the elastic deformation induced by a pressure distribution $p(x)$ acting on the elastic substrate. It can be computed as a convolution of the pressure with the Green's function of a line loading of a semi-infinite elastic half space. In terms of a scaling analysis, this becomes

$$
H \sim \frac{L P}{E}
$$

(deformation), where we anticipated that the dominant contribution comes from the integral over the pressure, over the dynamical horizontal scale $L$. Note that the size $L$ of this lubrication region will be much smaller than the total size of the contact $R_{c}$.

The outer shape corresponds to that of a static Hertz contact. In principle, this can be derived from (11), but here we simply pose the result, reading $h(x) \sim \frac{R_{c}^{1 / 2}|x|^{3 / 2}}{R}$, where $|x|$ is the distance measured

\footnotetext{
${ }^{1}$ Note that after the scraper has passed and the boundary condition changes from no slip to no stress, the thickness reduces to half this value.
} 


\section{JACCO H. SNOEIJER}

from the edge of the contact point [13]. In terms of scaling, this gives the matching condition

$$
H \sim \frac{R_{c}^{1 / 2} L^{3 / 2}}{R} .
$$

As before, the final step consists of combining (2), (12), and (13), yielding the scaling law

$$
H^{5} \sim \frac{(\eta U)^{3} R^{4}}{E^{3} R_{c}^{2}} .
$$

Further replacing the width of the contact zone $R_{c}$ by its value given in (10), we finally obtain

$$
H=0.45\left(\frac{\pi(3 \eta U R)^{3}\left(1-v^{2}\right)^{2}}{2 F E^{2}}\right)^{1 / 5},
$$

where the prefactor is drawn from the similarity analysis in [16]. Hence, the sought scaling law becomes $H \sim U^{3 / 5}$.

This completes the discussion of the three examples shown in Fig. 2, for which we have derived the scaling laws by following three times the same scheme.

\section{SOLID CAPILLARITY: ELASTIC INTERFACES WITH SURFACE TENSION}

We now turn to elastic interfaces in the limiting case of very low stiffness. Solid interfaces exhibit an excess surface free energy, just like liquid interfaces. However, only fairly recently has it been appreciated that the resulting surface tension $\gamma_{s}$, often called surface stress in solid mechanics [21,22,24], has important mechanical consequences: It implies a jump in normal stress $\gamma_{s} \kappa$, where $\kappa$ is the interface curvature, and this can dramatically change the mechanics of very soft elastic materials. Here we highlight a few striking consequences of solid surface tension.

\section{A. Solid Laplace pressure}

As the first example we consider the stability of thin cylindrical rods. Mora et al. [17] demonstrated that very soft cylinders exhibit a Rayleigh-Plateau-like instability driven by the solid's surface tension, in a way that is similar to liquid jets. Figure 4(a) from top to bottom shows agar gel cylinders of decreasing stiffness, the softer ones being unstable. Indeed, surface tension acts in the same manner as for liquids. Axisymmetric perturbations of the radius along the cylinder $r(z)=R\left(1+\epsilon e^{i k z}\right)$
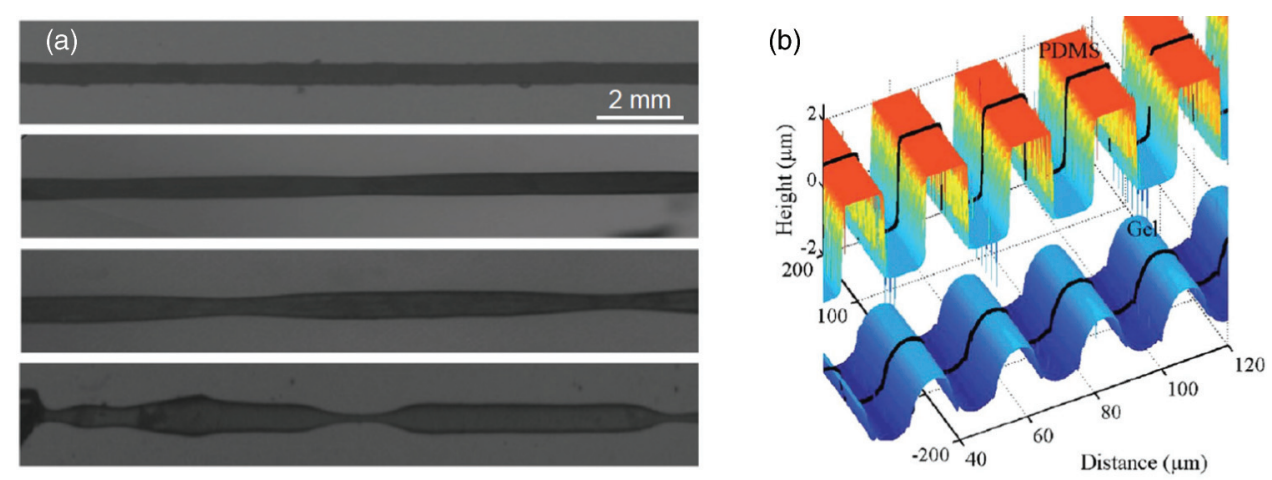

FIG. 4. Surface tension effects in elastic solids. (a) Thin cylinders of very soft agar gel can develop a Rayleigh-Plateau instability driven by the solid surface tension. The stiffness is decreased from top $(E=$ $81 \mathrm{~Pa}$ ) to bottom $(E=36 \mathrm{~Pa}$ ). (Reprinted with permission from [17]. Copyright 2010 by the American Physical Society.) (b) Rounding of sharp edges on a soft gelatine after it is withdrawn from a PDMS mould. (Reproduced from [19] with permission from the Royal Society of Chemistry.) 
induce a pressure jump

$$
\Delta P_{\gamma} \sim \epsilon \frac{\gamma_{s}}{R}\left[1-(k R)^{2}\right]
$$

where $R$ is the initial radius and $k$ is the wave number of the perturbation in the axial direction. For liquid jets the threshold for instability appears when $\Delta P_{\gamma}=0$ such that all waves with $k R<1$ are mechanically unstable. When the cylinder consists of a solid gel, however, the distortion of the interface induces an opposing elastic stress $\Delta P_{\mathrm{el}} \sim \epsilon E$, where $\epsilon=\delta r / R$ now provides the typical strain inside the soft solid gel. Balancing the capillary and elastic stress $\Delta P_{\gamma}=\Delta P_{\mathrm{el}}$ gives a marginal condition

$$
1-(k R)^{2} \sim \frac{E R}{\gamma_{s}},
$$

so instabilities can develop when the right-hand side is sufficiently small. Indeed, the detailed analysis and experiments in [17] show that the instability appears when $\gamma_{s} / E R>2$. In other words, the elastic cylinder is mechanically unstable when the ratio $\gamma_{s} / E$ exceeds the cylinder diameter.

The ratio $\gamma_{s} / E$ is the so-called elastocapillary length and sets the length scale below which capillary phenomena can compete with elasticity. This is further highlighted in the example in Fig. 4(b), taken from Paretkar et al. [19]. The figure shows the shape of a gelatine replica (lower image) after it is extracted from a stiff poly(dimethyl)siloxane (PDMS) mould (upper image). Clearly, the sharp crenellations of the mould are not present in the gelatine: All sharp features below the elastocapillary length are smoothened by surface tension. Within linear elasticity, one easily shows that sinusoidal perturbations of wave number $k$ are in fact reduced in amplitude by a factor $\left[1+\frac{3}{2} \frac{\gamma_{s} k}{E}\right]^{-1}$, once more illustrating the importance of the ratio $\gamma_{s} / E$.

\section{B. Soft wetting: Static and dynamic contact angles}

\section{Neumann's law}

The action of solid surface tension becomes even more pronounced when a liquid drop is deposited on a soft gel. The x-ray image in Fig. 5(a) from Park et al. [25] shows a detailed view of the three-phase contact line at the edge of a water drop. The solid is pinched by the action of the liquid-vapor surface tension $\gamma$. Importantly, the liquid-vapor interfacial forces are localized within a very narrow interfacial zone that extends over a nanometric scale $a$. In consequence, the liquid-vapor
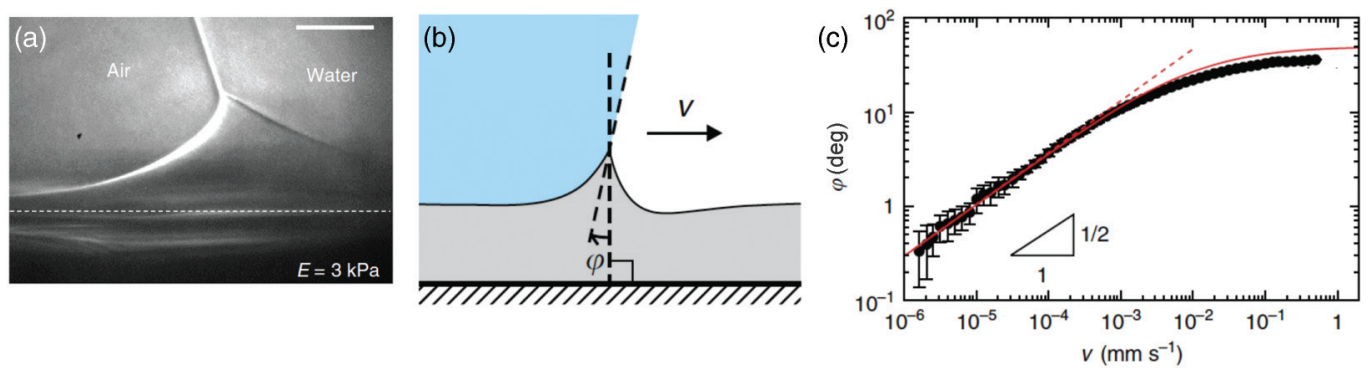

FIG. 5. Wetting a soft solid. (a) X-ray image of a three-phase contact line of a water drop on a soft silicon gel. The water drop induces substantial elastic deformation of the substrate. The scale bar indicates $5 \mu \mathrm{m}$. (Reprinted from [25], Nature Publishing Group, licensed under a Creative Commons Attribution 4.0 International Licence.) (b) Calculated profile of a wetting ridge that moves with a prescribed velocity $V$. The contact line motion induces a rotation of the wetting ridge by an angle $\varphi$. (c) Rotation angle $\varphi$ as a function of contact line velocity $V$, for water drops spreading on a silicon gel, comparing experiments (symbols) and theory (red solid line). [Panels (b) and (c) reprinted from [45], Nature Publishing Group, licensed under a Creative Commons Attribution 4.0 International Licence.] 


\section{JACCO H. SNOEIJER}

interface exerts a very large tensile stress on the solid that is of the order $\gamma / a \sim 10^{8} \mathrm{~Pa}$ [26-30]. For stiff materials such as glass (with $E \sim 10^{11} \mathrm{~Pa}$ ), such a stress does not induce any significant deformations and the substrate can be considered perfectly rigid, as is normally assumed in the derivation of Young's law for the contact angle. However, the stress induced by the liquid-vapor interface exceeds Young's modulus of the gel in Fig. 5(a) by at least four orders of magnitude. How does the gel resist such a large forcing near the contact line?

The presence of a solid surface tension can resolve this paradox [31-35]: A vectorial balance of the three surface tensions (solid-liquid, solid-vapor, and liquid-vapor) leads to the contact angles that satisfy Neumann's law. Indeed, the geometry of the contact line in Fig. 5(a) looks similar to that of liquid lenses, such as oil drops floating on water [11]. These observations are consistent with the discussion of the previous paragraphs, based on which one expects that surface tension dominates over elasticity at small scales, i.e., at distances smaller than $\gamma_{s} / E$. Whether or not the microscopic contact angles resemble that of Young's or Neumann's law then depends on whether the elastocapillary length $\gamma_{s} / E$ is small or large with respect to the microscopic size $a$ [32].

The emergence of Neumann's law as the relevant boundary condition for wetting on soft media has indeed been confirmed from detailed variational analysis, based on minimization of thermodynamic elastic and capillary energies [36]. However, we emphasize that this thermodynamic derivation crucially relied on the assumption that the surface free energy of the solid can be treated as a material constant that is independent of the elastic strain. A priori, such an approximation is not allowed for elastic interfaces, for which the free energy is not expected to remain constant when the material is strained. The potential consequences of this so-called Shuttleworth effect [37] are reviewed in [21].

\section{Dynamic contact angle}

Classically, the wetting and spreading dynamics of liquids over surfaces is described by a dynamic contact angle $\theta_{d}$ [38-40]. As the contact line moves with a velocity $V$, the dynamic (or apparent) angle of the liquid changes with respect to the equilibrium angle $\theta_{\mathrm{eq}}$. This nonequilibrium liquid angle correlates to the dissipation induced by the fluid motion. It was observed that the contact line speed is slowed down dramatically, by orders of magnitude, when the substrate is made exceedingly soft $[28,41,42]$. This led to the hypothesis that dissipation inside the substrate plays an important role, as also highlighted in recent experiments [43,44], since very soft materials typically are highly viscoelastic.

Karpitschka et al. [45] further showed how the dynamic contact angle can indeed be inferred directly from the viscoelastic dynamics inside the solid. The static wetting ridge was found to rotate by an angle $\varphi$ when the contact line is set into motion, as was computed numerically in Fig. 5(b). The amount of rotation depends strongly on the rheology of the solid. Figure 5(c) reports measurements of $\varphi=\theta_{d}-\theta_{\mathrm{eq}}$ as a function of contact line velocity $V$. At low velocity it is found that $\varphi \sim V^{0.55}$, which is very different from any classical wetting law. The origin of this scaling law can be traced back to the rheological spectrum: It reflects the scaling of the loss modulus of this material, which behaves as $G^{\prime \prime} \sim \omega^{0.55}$ at low frequency $\omega$. Indeed, a detailed theory based on the complete rheological spectrum of the solid leads to the red line shown in Fig. 5(c), capturing the full range of data [45]. Remarkably, since most of the dissipation occurs inside the substrate, the liquid can be considered at equilibrium during these spreading experiments.

\section{Adhesion of dry solids: Young's law}

We have seen that, on small scales, soft elastic interfaces develop wetting contact angles just like liquid interfaces. Does this also carry over when a gel is brought into contact with a rigid solid? Does the gel make a contact angle that satisfies Young's law? This question is relevant for indentation problems in contact mechanics, where a rigid sphere is brought into contact with an elastic layer, as shown in Fig. 6. In the presence of adhesion, such an indentation leads to the 

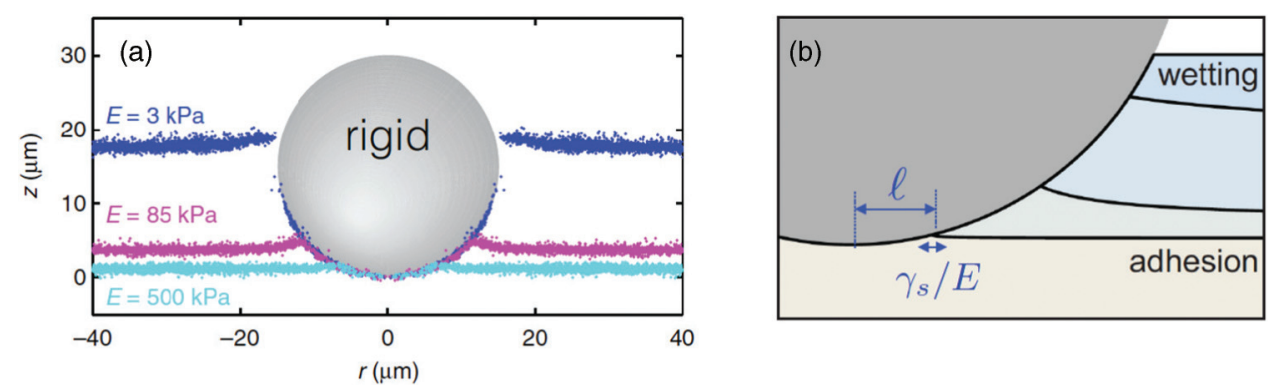

FIG. 6. Contact between a rigid indenter and a soft elastic layer. (a) Gels of varying stiffness indented by a rigid sphere, imaged by confocal microscopy. (Reprinted with permission from [48], Nature Publishing Group.) (b) Theoretical profiles of varying stiffness, including the definition of the contact width $\ell$. All profiles were computed with the very same Young angle at the three-phase contact line. [Image courtesy of Karpitschka (see [52] for details).] Stiff layers follow the classical JKR law (18) for solid adhesion $\left(\gamma_{s} / E \ll \ell\right)$, while soft layers behave as wetting liquids $\left(\gamma_{s} / E \gg \ell\right)$. These results suggest that Young's angle applies also for gels of finite stiffness.

classical Johnson-Kendall-Roberts (JKR) law for the width of the contact [46],

$$
\ell \sim\left(\frac{W R^{2}}{E}\right)^{1 / 3}
$$

valid when no external load is applied. Here $W$ is the work of adhesion and $R$ the radius of the indenter. We note that the derivation of this law relies on an analogy with fracture mechanics, for which the edge of the contact is treated as a singular crack [13,46,47]; hence, it remains unclear what happens at the contact line.

Recent work showed that it is indeed possible to extend the concept of contact angles to the adhesion of dry solids. An indication of this can be seen in the confocal microscopy images in Fig. 6(a) from Style et al. [48]. The softest gel (dark blue data) almost acts like a perfect liquid, in which the rigid sphere sinks until it reaches a desired angle. This wettinglike regime is not captured by the JKR law and emerges for very soft materials, when $\gamma_{s} / E \gg \ell$ [49-51]. In addition, Karpitschka et al. [52] investigated the boundary condition at the contact line by variational analysis. It was found that Young's law should in fact be satisfied not only for vanishing stiffness, but also for large $E$ (as long as $\gamma_{s} / E$ is larger than nanometric). The corresponding theoretical profiles for different stiffness are shown in Fig. 6(b). These profiles capture the trends observed experimentally and are in perfect agreement with the classical law (18) at large stiffness. Though not visible in Fig. 6(b), all profiles exhibit the very same contact angle as a boundary condition. This series of observations provide a unification between the adhesion of dry solids and the wetting of liquids: The difference is only in the relative strength of elasticity and capillarity.

\section{DISCUSSION}

This paper explored the possible merits of drawing analogies between elastic and capillary interfacial flows. We first set side by side three apparently distinct lubrication problems and solved them by applying a single method. We then showed that very soft elastic surfaces in fact behave very similarly to liquid interfaces, owing to their surface tension. Perhaps the most remarkable feature is that soft solids can be folded into liquidlike contact angles when in contact with a liquid or another solid. Based on this, one can appreciate a deep connection between liquid wetting and the mechanics of adhesive solids: Both represent different limiting cases of an adhesion problem, governed by the very same wetting boundary condition. 


\section{JACCO H. SNOEIJER}

It is tempting to consider the elastic-capillary analogy between other types of lubrication problems such as the dimple formation during normal impact shown in Fig. 1. Similarly, the spreading of droplets under elastic membranes gives rise to interesting dynamics reminiscent of capillary drop spreading [53], and many other examples could be given. A particularly nice example is found for the Cheerios effect [54], i.e., the interaction of particles at a fluid interface, for which elastic counterparts were proposed recently. Solid particles were found to attract when deforming elastic interfaces by their weight $[55,56]$. The roles of solid and liquid were even completely reversed in the inverted Cheerios effect, where liquid drops on soft solids were also found to interact by the induced elastic deformation [57]. Surprisingly, the interaction between droplets could be tuned from attractive to repulsive upon varying the thickness of the gel layer. Exploiting the elastic-capillary analogy hence continues to be a fruitful approach for a broad variety of interfacial phenomena.

\section{ACKNOWLEDGMENTS}

I am indebted to many collaborators. In particular, I would like to thank Bruno Andreotti, Siddhartha Das, Jens Eggers, Kees Venner, Stefan Karpitschka, Mathijs van Gorcum, and Anupam Pandey for carefully reading the manuscript. Financial support from the European Research Council Consolidator Grant No. 616918 is acknowledged.

[1] R. C. A. van der Veen, T. Tran, D. Lohse, and C Sun, Direct measurements of air layer profiles under impacting droplets using high-speed color interferometry, Phys. Rev. E 85, 026315 (2012).

[2] Y. Wang, C. Dhong, and J. Frechette, Out-of-Contact Elastohydrodynamic Deformation due to Lubrication Forces, Phys. Rev. Lett. 115, 248302 (2015).

[3] R. H. Davis, J. M. Serayssol, and E. J. Hinch, The elastohydrodynamic collision of two spheres, J. Fluid Mech. 163, 479 (1986).

[4] L. G. Leal, Advanced Transport Phenomena: Fluid Mechanics and Convective Transport Processes (Cambridge University Press, Cambridge, 2007).

[5] S. Mandre, M. Mani, and M. P. Brenner, Precursors to Splashing of Liquid Droplets on a Solid Surface, Phys. Rev. Lett. 102, 134502 (2009).

[6] S. Leroy, A. Steinberger, C. Cottin-Bizonne, F. Restagno, L. Leger, and E. Charlaix, Hydrodynamic Interaction between a Spherical Particle and an Elastic Surface: A Gentle Probe for Soft thin Films, Phys. Rev. Lett. 108, 264501 (2012).

[7] W. Bouwhuis, R. C. A. van der Veen, T. Tran, D. L. Keij, K. G. Winkels, I. R. Peters, D. van der Meer, C. Sun, J. H. Snoeijer, and D. Lohse, Maximal Air Bubble Entrainment at Liquid-Drop Impact, Phys. Rev. Lett. 109, 264501 (2012).

[8] F. P. Bretherton, The motion of long bubbles in tubes, J. Fluid Mech. 10, 166 (1961).

[9] L. D. Landau and B. V. Levich, Dragging of a liquid by a moving plate, Acta Phys. Chim. USSR 17, 42 (1942).

[10] K. J. Mysels, K. Shinoda, and S. Frankel, Soap Films: Studies of their Thinning and a Bibliography (Pergamon, Oxford, 1959).

[11] P.-G. de Gennes, F. Brochard-Wyart, and D. Quere, Capillarity and Wetting Phenomena: Drops, Bubbles, Pearls, Waves (Springer, New York, 2004).

[12] C. J. Hooke and J. P. O’Donoghue, Elastohydrodynamic lubrication of soft, highly deformed contacts, J. Mech. Eng. Sci. 14, 34 (1972).

[13] K. L. Johnson, Contact Mechanics (Cambridge University Press, Cambridge, 1985).

[14] D. Dowson, History of Tribology, 2nd ed. (Wiley, New York, 1998).

[15] C. H. Venner and A. A. Lubrecht, MultiLevel Methods in Lubrication, edited by D. Dowson, Tribology Series Vol. 37 (Elsevier, Amsterdam, 2000). 
[16] J. H. Snoeijer, J. Eggers, and C. H. Venner, Similarity theory of lubricated Hertzian contacts, Phys. Fluids 25, 101705 (2013).

[17] S. Mora, T. Phou, J.-M. Fromental, L. M. Pismen, and Y. Pomeau, Capillarity Driven Instability of a Soft Solid, Phys. Rev. Lett. 105, 214301 (2010).

[18] S. Mora, C. Maurini, T. Phou, J.-M. Fromental, B. Audoly, and Y. Pomeau, Solid Drops: Large Capillary Deformations of Immersed Elastic Rods, Phys. Rev. Lett. 111, 114301 (2013).

[19] D. Paretkar, X. Xu, C. Y. Hui, and A. Jagota, Flattening of a patterned compliant solid by surface stress, Soft Matter 10, 4084 (2014).

[20] B. Andreotti, O. Baumchen, F. Boulogne, K. E. Daniels, E. R. Dufresne, H. Perrin, T. Salez, J. H. Snoeijer, and R. W. Style, Solid capillarity: When and how does surface tension deform soft solids? Soft Matter 12, 2993 (2016).

[21] B. Andreotti and J. H. Snoeijer, Soft wetting and the Shuttleworth effect, at the crossroads between thermodynamics and mechanics, Europhys. Lett. 113, 66001 (2016).

[22] R. W. Style, A. Jagota, C.-Y. Hui, and E. R. Dufresne, Elastocapillarity: Surface tension and the mechanics of soft solids, arXiv:1604.02052.

[23] J. Seiwert, D. Quéré, and C. Clanet, Flexible scraping of viscous fluids, J. Fluid Mech. 715, 424 (2013).

[24] P. Müller and A. Saúl, Elastic effects on surface physics, Surf. Sci. Rep. 54, 157 (2004).

[25] S. J. Park, B. M. Weon, J. S. Lee, J. Lee, J. Kim, and J. H. Je, Visualization of asymmetric wetting ridges on soft solids with X-ray microscopy, Nat. Commun. 5, 4369 (2014).

[26] J. S. Rowlinson and B. Widom, Molecular Theory of Capillarity (Clarendon, Oxford, 1982).

[27] A. I. Rusanov, Thermodynamics of deformable solid-surfaces, J. Colloid Interface Sci. 63, 330 (1978).

[28] A. Carre, J. C. Gastel, and M. E. R. Shanahan, Viscoelastic effects in the spreading of liquids, Nature (London) 379, 432 (1996).

[29] L. R. White, The contact angle on an elastic substrate. 1. The role of disjoining pressure in the surface mechanics, J. Colloid Interface Sci. 258, 82 (2003).

[30] R. Pericet-Camara, E. Bonaccurso, and K. Graf, Microstructuring of polystyrene surfaces with nonsolvent sessile droplets, Chem. Phys. Chem. 9, 1738 (2008).

[31] E. R. Jerison, Y. Xu, L. A. Wilen, and E. R. Dufresne, Deformation of an Elastic Substrate by a Three-Phase Contact Line, Phys. Rev. Lett. 106, 186103 (2011).

[32] A. Marchand, S. Das, J. H. Snoeijer, and B. Andreotti, Contact Angles on a Soft Solid: From Young's Law to Neumann's Law, Phys. Rev. Lett. 109, 236101 (2012).

[33] L. Limat, Straight contact lines on a soft, incompressible solid, Eur. Phys. J. E 35, 134 (2012).

[34] R. W. Style, R. Boltyanskiy, Y. Che, J. S. Wettlaufer, L. A. Wilen, and E. R. Dufresne, Universal Deformation of Soft Substrates Near a Contact Line and the Direct Measurement of Solid Surface Stresses, Phys. Rev. Lett. 110, 066103 (2013).

[35] J. B. Bostwick, M. Shearer, and K. E. Daniels, Elastocapillary deformations on partially-wetting substrates: Rival contact-line models, Soft Matter 10, 7361 (2014).

[36] L. A. Lubbers, J. H. Weijs, L. Botto, S. Das, B. Andreotti, and J. H. Snoeijer, Drops on soft solids: Free energy and double transition of contact angles, J. Fluid. Mech. 747, R1 (2014).

[37] R. Shuttleworth, The surface tension of solids, Proc. Phys. Soc. London Sect. A 63, 444 (1950).

[38] P.-G. de Gennes, Wetting: Statics and dynamics, Rev. Mod. Phys. 57, 827 (1985).

[39] D. Bonn, J. Eggers, J. Indekeu, J. Meunier, and E. Rolley, Wetting and spreading, Rev. Mod. Phys. 81, 739 (2009).

[40] J. H. Snoeijer and B. Andreotti, Moving contact lines: Scales, regimes, and dynamical transitions, Annu. Rev. Fluid Mech. 45, 269 (2013).

[41] M. E. R. Shanahan and A. Carre, Viscoelastic dissipation in wetting and adhesion phenomena, Langmuir 11, 1396 (1995).

[42] D. Long, A. Ajdari, and L. Leibler, Static and dynamic wetting properties of thin rubber films, Langmuir 12, 5221 (1996).

[43] T. Kajiya, A. Daerr, T. Narita, L. Royon, F. Lequeux, and L. Limat, Advancing liquid contact line on visco-elastic gel substrates: Stick-slip vs. continuous motions, Soft Matter 9, 454 (2013). 


\section{JACCO H. SNOEIJER}

[44] T. Kajiya, P. Brunet, L. Royon, A. Daerr, M. Receveur, and L. Limat, A liquid contact line receding on a soft gel surface: Dip-coating geometry investigation, Soft Matter 10, 8888 (2014).

[45] S. Karpitschka, S. Das, M. van Gorcum, H. Perrin, B. Andreott, and J. H. Snoeijer, Droplets move over viscoelastic substrates by surfing a ridge, Nat. Commun. 6, 7891 (2015).

[46] K. L. Johnson, K. Kendall, and A. D. Roberts, Surface energy and the contact of elastic solids, Proc. R. Soc. London A 324, 301 (1971).

[47] D. Maugis and M. Barquins, Fracture mechanics and adherence of viscoelastic bodies, J. Phys. D: Appl. Phys. 11, 1989 (1978).

[48] R. W. Style, C. Hyland, R. Boltyanskiy, J. S. Wettlaufer, and E. R. Dufresne, Surface tension and contact with soft elastic solids, Nat. Commun. 4, 2728 (2013).

[49] T. Salez, M. Benzaquen, and E. Raphael, From adhesion to wetting of a soft particle, Soft Matter 9, 10699 (2013).

[50] Z. Cao, M. J. Stevens, and A. V. Dobrynin, Adhesion and wetting of nanoparticles on soft surfaces, Macromolecules 47, 3203 (2014).

[51] T. Liu, A. Jagota, and C.-Y. Hui, Adhesive contact of a rigid circular cylinder to a soft elastic substrate-The role of surface tension, Soft Matter 11, 3844 (2015).

[52] S. Karpitschka, L. van Wijngaarden, and J. H. Snoeijer, Surface tension regularizes the crack singularity of adhesion, Soft Matter 12, 4463 (2016).

[53] J. R. Lister, G. G. Peng, and J. A. Neufeld, Viscous Control of Peeling an Elastic Sheet by Bending and Pulling, Phys. Rev. Lett. 111, 154501 (2013).

[54] D Vella and L Mahadevan, The Cheerios effect, Am. J. Phys. 73, 817 (2005).

[55] A. Chakrabarti and M. Chaudhury, Elastocapillary interaction of particles on the surfaces of ultrasoft gels: A novel route to study self-assembly and soft lubrication, Langmuir 30, 4684 (2014).

[56] A. Chakrabarti, L. Ryan, M. Chaudhury, and L. Mahadevan, Elastic cheerios effect: Self-assembly of cylinders on a soft solid, EPL 112, 54001 (2015).

[57] S. Karpitschka, A. Pandey, L. A. Lubbers, J. H. Weijs, L. Botto, S. Das, B. Andreotti, and J. H. Snoeijer, Liquid drops attract or repel by the inverted Cheerios effect, Proc. Natl. Acad. Sci. USA 113, 7403 (2016). 